\title{
Analysis of Parental Decisions Based on Sex Chromo- some Abnormalities Detected Prenatally: A Ten-year up- date (2001-2010)
}

Gye-Hyeong An ${ }^{1}$, Kyu-Hong Choi', Jae-Hyug Yang ${ }^{1}$, Moon-Young Kim ${ }^{1}$, Jung-Yeol Han', So-Yeon Park ${ }^{2}$, Bom-Yi Lee ${ }^{2}$, Da-Eun Lee and Hyun-Mee $\mathrm{Ryu}^{1,2^{*}}$

'Department of Obstetrics and Gynecology, Cheil General Hospital and Women's Healthcare Center, Kwandong University College of Medicine, Seoul, Korea

²Laboratory of Medical Genetics, Medical Research Institute, Cheil General Hospital and Women's Healthcare Center, Seoul, Korea

Purpose: The aim of this study was to analyze parental decisions regarding pregnancies in which the fetus had sex chromosome abnormalities (SCA) over a ten-year period.

Materials and Methods: We collected and reviewed records from our hospital for 2001-2010 and a genetic specialist providedgenetic counseling.

Results: We diagnosed 130 cases $(0.71 \%)$ with SCA out of 18,376 prenatal cases from 2001 to 2010 . We reviewed the records and the results of all pregnancies. We also included cases $(n=84)$ of apparently normal anatomic fetuses to analyze the factors influencing parental decisions. We excluded 34 cases with an obvious anomaly or a presumably bad outcome and 12 cases that were not followed up. Forty-three couples (51.2\%) continued their pregnancies while forty-one (48.8\%) terminated them. Of 38 mosaicism cases, 21 (55.3\%) were continued. Among the 20 pregnancies assisted by reproductive techniques, 15 (75\%) were continued $(P=0.02)$. More pregnancies were continued when genetic counseling was provided $(61.9 \%)$ compared to cases in which it was not provided $(19 \%)(P=0.01)$.

Conclusion: Genetic counseling is important in providing appropriate information to parents. Establishing guidelines and protocols will help both obstetricians and parents to make informed decisions.

Key Words: Sex chromosome aberration, Prenatal diagnosis, Genetic counseling

\section{Introduction}

A prenatal diagnosis is offered to couples with an increased risk of genetic abnormalities. Prenatal detection of sex chromosome abnormalities is increasing due to the widespread use of prenatal diagnostic procedures such as amniocentesis, chorionic villus sampling and cordocentesis for karyotyping.

Sex chromosome aneuploidy is defined as a numeric abnormality of an X or $Y$ chromosome such as 45, X (Turner syndrome), 47,XXX (Triple $X$ syndrome), 47,XXY (Klinefelter syndrome), and 47,XYY. Mosaic and structural variants of chromosomes $X$ and $Y$ are also considered to be sex chromosome aneuploidy.

Received: 31 May 2012, Revised: 20 June 2012, Accepted: 21 June 2012, Published: 30 June 2012

${ }^{*}$ Corresponding author: Hyun Mee Ryu, M.D., Ph.D.

Department of Obstetrics and Gynecology, Cheil General Hospital and Women's Healthcare Center, Kwandong University College of Medicine, 1-19 Mukjeong-dong, Jung-gu, Seoul 100-380, Korea

Tel: +82-2-2000-7683, Fax: +82-2-2278-4574, E-mail: hmryu@yahoo.com

(c) This is an open-access article distributed under the terms of the Creative Commons Attribution Non-Commercial License (http://creativecommons.org/licenses/by-nc/3.0/) which permits unrestricted non-commercial use, distribution, and reproduction in any medium, provided the original work is properly cited.

(c) Copyright 2012 by the Korean Society of Medical Genetics www.e-kjgm.org 
Major malformations may occur in some Turner syndrome cases and these are associated with about 80\% prenatal loss and high postnatal morbidity. ${ }^{1,2)}$ Men with Klinefelter syndrome generally show infertility and tall stature, but they have no severe impairment in intelligence or behavior. ${ }^{3)}$ Also, most cases of Klinefelter, Triple $X_{\text {, }}$ and $X Y Y$ syndromes have a normal life expectancy and patients have well adjusted social lives. Mosaic patients are affected mildly or are phenotypically normal, compared with non-mosaics. ${ }^{4}$

Despite the relatively good prognosis of sex chromosome aneuploidy, most parents feel uncertain with continuing their pregnancy if they receive such a diagnosis. This is usually due to the fear of reduced intelligence, learning and behavioral problems, tall or short stature and the possibility of having a disabled child. However, the intelligence of children with SCA has shown to be average or above average at school, if they have had good home education and counseling.

Previous studies have shown that a major proportion of SCA pregnancies are terminated. ${ }^{6,7)}$ However, recent studies suggest that careful genetic counseling can provide more information to the parents and reduce the termination rate of such pregnancies. ${ }^{8-12)}$

In the present retrospective study, we reviewed SCA cases that were diagnosed prenatally over the past ten years at Cheil General Hospital in Korea. In this time period prenatal karyotyping was done in 18,376 cases. We found 130 cases with SCA and performed a retrospective analysis of the postcounseling outcomes of these cases.

The purpose of this study was to analyze parental decisions regarding pregnancies with SCA over ten years. Potential factors influencing parental decisions to continue or terminate such a pregnancy were also analyzed.

\section{Materials and Methods}

Between 2001 and 2010, we diagnosed 130 cases (0.71\%) of SCA out of 18,376 prenatal cases. The diagnosis of all cases with SCA (karyotypes 45, $X, 47, X X Y, 47, X X X$, and 47,XYY in a non-mosaic or mosaic state) was made by examination of the original patient charts and laboratory data sheets.

Diagnostic methods included amniocentesis and cordocentesis. We included cases ( $n=84)$ of apparently anatomically normal fetuses to analyze the factors influencing parental decisions. We excluded 34 cases with obvious anomalies or presumably bad outcomes such as non-mosaic Turner syndrome, because it is commonly associated with fetal hydrops and/or cystic hygroma.
Additionally, a case of 47,XXY with multiple anomalies was excluded, as was a case of $48, X X X X$ and 12 cases that were lost to follow-up. After excluding all 46 of these cases, the remaining 84 cases were analyzed.

The karyotypes of patients with 47,XXY, 47,XXX, and 47,XYY in a non-mosaic or mosaic state were included. Mosaic Turner syndromes were also included. However, cases of presumed pseudo-mosaicism were excluded because of culture artifacts or maternal cell contamination.

Families were counseled by ten different obstetricians and the obstetricians recommended further genetic counseling with MD geneticists. Families were informed of the characteristics, prognosis, management and possible treatment options for the associated abnormalities. They were generally informed that individuals with SCA have a normal phenotype and that individuals with Klinefelter syndrome may have a tall stature, could develop gynecomastia and are usually infertile, but have no sexual problems. Individuals with $47, X X X$ and $47, X Y Y$ are often taller than average, but usually do not have other clinical manifestations. Families were informed that individuals with SCA have normal intelligence, but they may have a slightly lower IQ than their siblings. When the result was a mosaic pattern, the families were counseled regarding a wide spectrum of features ranging from a normal phenotype to classic features of the specific SCA. Individuals with a mosaic $45, \mathrm{X}$ may have a short stature, cardiac problems, primary amenorrhea, lack of secondary sexcharacteristics, and may be infertile.

In cases with 45,X/46,XY, the possibility of mixed gonadal dysgenesis was also explained to the parents. The families were generally told that $95 \%$ of pregnancies with a prenatal diagnosis of mixed gonadal dysgenesis result in a normal phenotypic male with a possibility of infertility and an increased risk of gonadal tumors. The remaining 5\% of the pregnancies result in an infant with some degree of ambiguous genitalia, which may require surgery, or a normal phenotypic female with some Turner syndrome features. ${ }^{11,12)}$

The courses and outcomes of the pregnancies were reviewed from the patients' charts. The genetic counseling, assisted reproductive technology procedure, the presence or absence of a fetal structural anomaly on ultrasonographic examination, the type of SCA, and the prenatal procedure performed were reviewed. We also analyzed the pregnancy continuation rate by temporal period. The outcomes of ten babies delivered at other hospitals were confirmed by telephone interviews. Statistical analysis was performed via Fisher's exact test. The differences were deemed significant when $P$ was $<0.05$. 


\section{Results}

A total of 84 pregnancies with a SCA diagnosis were included in our analysis. These included 19 cases of $47, X X Y, 14$ cases of $47, X X X, 12$ cases of $47, X Y Y$, and 38 cases of several types of mosaicism and 1 case of $47, X Y, i(X)(q 10)$. We found that 79 cases were single pregnancies and 5 cases were twins whose karyotypes were $47, X Y Y, 47, X X Y$, and $47, X X X$, and 2 cases of $45, X$ mosaicism, while the other twins had normal karyotypes. All five twin pregnancies were continued. The diagnoses were made between the 15.3th and 25.2th gestational weeks and were confirmed or corrected by an early gestational ultrasonogram.

For prenatal testing, 77 (91.7\%) women underwent amniocentesis, 3 (3.6\%) underwent cordocentesis and 4 (4.8\%) underwent amniocentesis confirmed by cordocentesis (Table 1). However, a skin biopsy of the fetus was not performed. The mean gestational age at the time the invasive procedures were performed was 17.7 weeks. Indications included advanced maternal age ( $>35$ years) $(n=40)$, abnormal maternal serum screen $(n=22)$, abnormal sonographic findings (increased fetal nuchal translucency, $n=12$ ), abnormal maternal serum screen with AMA ( $n=2)$, abnormal sonographic findings with AMA $(n=7)$, and pregestational diagnoses (PGD) ( $n=1)$ (Table 2).

Table 3 summarizes the cytogenetic findings and parental decisions of the 84 analyzed cases with SCA. The results show that 41 couples (48.8\%) terminated their pregnancies and 43 (51.2\%) continued their pregnancies. Also, 21 couples (55.3\%) out of 38 mosaicism cases continued their pregnancies and there was no statistical significance ( $P=0.44$ ) (Table 4).

Table 1. Methods for prenatal testing

\begin{tabular}{lc}
\hline Diagnostic method & Number of cases \\
\hline Amniocentesis & 77 \\
Cordocentesis & 3 \\
Amniocentesis and cordocentesis & 4 \\
Total & 84 \\
\hline
\end{tabular}

Table 2. Indications for prenatal testing

\begin{tabular}{lc}
\hline Indications for prenatal testing & Number of cases \\
\hline Advanced maternal age (AMA) & 40 \\
Serum screening & 22 \\
Ultrasound screening & 12 \\
AMA and serum screening & 2 \\
AMA and ultrasound screening & 7 \\
Pregestational diagnosis & 1 \\
Total & 84
\end{tabular}

Among the 84 fetuses with SCA were 46 non-mosaic and 38 mosaic cases. Of the mosaic cases, 31 were $45, \mathrm{X}$ mosaicism and $54.8 \%$ of these pregnancies were continued. Four couples (57.1\%) out of the seven other mosaic pregnancies were continued (Table 5). Of the 20 couples who underwent assisted reproductive techniques, 15 couples $(75 \%)$ continued their pregnancies $(P=0.023)$ (Table 6).

We found that parental decisions differed depending on the period in which the prenatal SCA diagnoses were made. A relatively higher proportion of couples chose to continue their pregnancies in recent years (Table 7). More SCA pregnancies were continued when couples participated in counseling administered by a MD geneticist. Among the 63 couples that received genetic counseling, 39 continued their

Table 3. Cytogenetic diagnoses and outcomes in 84 pregnancies with sex chromosome abnormalities

\begin{tabular}{lccc}
\hline Karyotype & Cases $(n)$ & Continued $(n)$ & $\%$ \\
\hline $47, X X Y$ & 19 & 7 & 36.8 \\
$47, X X X$ & 14 & 6 & 42.9 \\
$47, X Y Y$ & 12 & 8 & 66.7 \\
$45, X / 46, X X$ & 10 & 4 & 40 \\
$45, X / 46, X Y$ & 15 & 9 & 60 \\
$45, X / 47, X X X$ & 4 & 3 & 75 \\
$45, X / 47, X Y Y$ & 2 & 1 & 50 \\
$46, X X / 47, X X X$ & 2 & 1 & 50 \\
$46, X Y / 47, X X Y$ & 1 & 1 & 100 \\
$46, X Y / 47, X Y Y$ & 2 & 0 & 0 \\
$47, X X Y / 45, X / 46, X Y$ & 1 & 1 & 100 \\
$46, X X / 46, X Y$ & 1 & 1 & 100 \\
$47, X Y, i(X)(q 10)$ & 1 & 1 & 100 \\
Total & 84 & 43 & 51.2 \\
\hline
\end{tabular}

Table 4. Parental decisions and mosaicism

\begin{tabular}{lccc}
\hline Mosaicism & Cases $(n)$ & Continued $(n)$ & $\%$ \\
\hline Non-Mosaic & 46 & 22 & 47.8 \\
Mosaic & 38 & 21 & 55.3 \\
Total & 84 & 43 & 51.2 \\
\hline
\end{tabular}

${ }^{*} P$-value $=0.44$ (Fisher's exact test)

Table 5. Cytogenetic diagnoses and outcomes in 84 pregnancies with sex chromosome abnormalities

\begin{tabular}{lccc}
\hline Karyotype & Cases $(n)$ & Continued $(n)$ & $\%$ \\
\hline 47, XXY & 19 & 7 & 36.8 \\
47, XXX & 14 & 6 & 42.9 \\
47, XYY & 12 & 8 & 66.7 \\
45, X mosaicism & 31 & 17 & 54.8 \\
Other mosaicism & 7 & 4 & 57.1 \\
$47 X Y, i(X)(q 10)$ & 1 & 1 & 100 \\
Total & 84 & 43 & 51.2
\end{tabular}


pregnancy (61.9\%). Of the 21 cases that did not receive genetic counseling, only 4 couples continued their pregnancies (19\%). The difference in the number of continued pregnancies between these two groups was statistically significant $(P=0.001)$ (Table 8). We further stratified the cases who received genetic counseling by SCA type. However, there were no significant differences in parental decision-making between subgroups (Table 9). Our data does show that genetic counseling was helpful for parental decision making with regard to the continuation of SCA pregnancies.

\section{Discussion}

SCA is the most frequently encountered chromosomal abnormality both prenatally and at birth. ${ }^{5)}$ Approximately 1 out of every 400 newborns has an SCA, comprising $25 \%$ of all cases of chromosomal abnormalities. ${ }^{13,14)}$

Table 6. Parental decisions and reproductive history

\begin{tabular}{lccc}
\hline Type & Total $(\mathrm{n})$ & Continued $(\mathrm{n})$ & $\%$ \\
\hline Natural pregnancy & 64 & 28 & 43.8 \\
Assisted reproductive technology & 20 & 15 & 75 \\
Total & 84 & 43 & 51.2 \\
\hline
\end{tabular}

${ }^{*} P$-value $=0.023$ (Fisher's exact test)

Table 7. Parental decisions by time period

\begin{tabular}{lccc}
\hline Years & Continued $(n)$ & $\%$ & Total $(n)$ \\
\hline $2001-2004$ & 15 & 46.9 & 32 \\
$2005-2007$ & 12 & 48 & 25 \\
$2008-2010$ & 16 & 59.3 & 27 \\
Total & 43 & 51.2 & 84 \\
\hline
\end{tabular}

Table 8. Parental decisions and genetic counseling

\begin{tabular}{lccccc} 
& Cases (n) & Terminated (n) & $\%$ & Continued (n) & $\%$ \\
\hline Genetic counseling & 63 & 24 & 38.1 & 39 & 61.9 \\
No genetic counseling & 21 & 17 & 81 & 4 & 19 \\
Total & 84 & 41 & 48.8 & 43 & 51.2 \\
\hline
\end{tabular}

${ }^{\star} P$-value $=0.001$ (Fisher's exact test)
With an increasing number of pregnancies being subjected to prenatal karyotyping because of abnormal ultrasound findings and positive maternal serum screening results, it is inevitable that a large proportion of fetuses with SCA are detected prenatally. As a result, parents are faced with a very difficult and personal decision when a SCA is identified.

Our study showed that $51.2 \%$ of couples at our center continued their pregnancy following a prenatal diagnosis of SCA, excluding cases of non-mosaic Turner syndrome.

Most reports from other institutions present termination rates between 32\% and 66\%. ${ }^{15)}$ Mahmut et al. (2010) reported that among the couples with normal fetal ultrasound findings, the overall continuation rate was $60 \% .{ }^{16)}$ A significant decrease in the pregnancy termination rate, from 100\% in 1976-1979 to $54 \%$ in 1995-1997, was found in Canada. ${ }^{17)}$ An unusually low termination rate was reported for Munster. ${ }^{18}$ Sheng-Wen etal. (2008) reported that among 57 fetuses with SCA (36 non-mosaic cases and 21 mosaic cases, including 15 45,X mosaicism) only 20\% of the $45, X$ mosaic pregnancies were continued, whereas all other mosaic pregnancies $(100 \%)$ were continued $(P=0.004))^{19)}$

In the present study, the decision by parents to continue or terminate a pregnancy with SCA was dependent on many factors including genetic counseling, infertility, the presence or absence of mosaicism, and the type of SCA. In our study, $42.9 \%$ of pregnancies with $47, X X X$ and $36.8 \%$ of pregnancies with 47,XXY were continued (Table 5). The parents' perception of the expected disability was believed to play an important role in deciding whether to continue or terminate. ${ }^{20)}$ Although the presence or absence of mosaicism did not significantly influence the couples' decisions regarding their pregnancy (Table 4), the data suggests that couples were more likely to continue their pregnancy with a mosaic karyotype. The temporal period analysis showed a tendency toward a slight increase in the continuation rate over time. The trend toward a higher rate of continuation may be a consequence of the publication of studies on long-term prospective outcomes in both newborns and pregnancies diagnosed with SCA. ${ }^{211}$

The decision to terminate a pregnancy due to a diagnosis of

Table 9. Karyotype and genetic counseling

\begin{tabular}{|c|c|c|c|c|c|c|c|}
\hline \multirow{2}{*}{ Karyotype } & \multicolumn{3}{|c|}{ Genetic counseling } & \multicolumn{3}{|c|}{ No genetic counseling } & \multirow{2}{*}{$P$-value } \\
\hline & Cases (n) & Continued (n) & $(\%)$ & Cases (n) & Continued (n) & $(\%)$ & \\
\hline $47, X X X$ & 12 & 6 & 50 & 2 & 0 & 0 & 0.47 \\
\hline $47, X X Y$ & 11 & 6 & 54.5 & 8 & 1 & 12.5 & 0.15 \\
\hline $47, X Y Y$ & 11 & 8 & 72.7 & 1 & 0 & 0 & 0.33 \\
\hline $45, X / 46, X X$ & 7 & 4 & 57.1 & 3 & 0 & 0 & 0.20 \\
\hline $45, X / 46, X Y$ & 11 & 8 & 72.7 & 4 & 1 & 25 & 0.24 \\
\hline
\end{tabular}

${ }^{\star} P$-value (Fisher's exact test) 
SCA may be related in part to genetic counseling strategies. In our study, more pregnancies with SCA were continued when the post-diagnosis counseling was administered by an MD geneticist compared to when parents did not receive counseling (Table 8). Thus, it is essential for an obstetric unit to have an established protocol for discussing results and for all staff members who communicate with parents to give accurate, up-to-date information about the identified condition.

Although the present study did not detect a significantly low pregnancy termination rate (48.8\%), it is low compared to previous studies. This finding may be due to the genetic counseling parents received. Thus, genetic counseling by well-trained specialists is important to ensure that appropriate information is given to parents. Establishing guidelines and protocols will help both obstetricians and parents to make an informed decision. It appears that the development of consensus guidelines in pluridisciplinary fetal care centers would help reduce disparities in the management of fetuses with SCA.

\section{Acknowledgment}

This study was supported by a grant of the Korean Health Technology R\&D Project, Ministry of Health \& Welfare, Republic of Korea (A111550).

\section{References}

1. Bender $B G$, Linden MG, Robinson A. Neuropsychological impairment impairment in 42 adolescents with sex chromosome abnormalities. Am J Med Genet 1993;48:69-73.

2. Abramsky L, Chapple J. 47,XXY (Klinefelter syndrome) and 47,XYY: estimated rates of and indication for postnatal diagnosis with implications for prenatal counselling. Prenat Diagn 1997;17:363-8.

3. Hall JG, Gilchrist DM. Turner syndrome and its variant. Pediatr Clin North Am 1990;37:1421-40.

4. Bender B, Fry E, Pennington B, Puck M, Salbenblatt J, Robinson A. Speech and language development in 41 children with sex chromosome anomalies. Pediatrics 1983;71:262-7.

5. Abramsky L, Hall S, Levitan J, Marteau TM. What parents are told after prenatal diagnosis of a sex chromosome abnormality: interview and questionnaire study. BMJ 2001;322:463-6.

6. Nielsen J. Follow-up of 25 unselected children with sex chromosome abnormalities to age 12. Birth Defects Orig Artic Ser 1990;26:201-7.
7. Linden $\mathrm{MG}$, Bender $\mathrm{BG}$, Robinson $\mathrm{A}$. Intrauterine diagnosis of sex chromosome aneuploidy. Obstet Gynecol 1996;87:468-75.

8. Christian SM, Koehn D, Pillay R, MacDougall A, Wilson RD. Parental decisions following prenatal diagnosis of sex chromosome abnormality: a trend over time. Prenat Diagn 2000;20:37-40.

9. Evans JA, MacDonald K, Hamerton JL. Sex chromosome anomalies: prenatal diagnosis and the need for continued prospective studies. Birth Defects Orig Artic Ser 1990;26:273-81.

10. Meschede D, Louwen F, Nippert I, Holzgreve W, Miny P, Horst J. Low rates of pregnancy termination for prenatally diagnosed sex chromosome polysomies. Am J Med Genet 1998;80:330-4.

11. Chang HJ, Clark RD, Bachman $H$. The phenotype of $45, X / 46, X Y$ mosaicism: an analysis of 92 prenatally diagnosed cases. Am J Hum Genet 1990;46:156-67.

12. Robinson A, Bender BG, Linden MG. Prognosis of prenatally diagnosed children with sex chromosome aneuploidy. Am J Med Genet 1992;44: 365-8.

13. Jacobs PA. The incidence and etiology of sex chromosome abnormalities in man. Birth Defects Orig Artic Ser 1979;15:3-14.

14. Cradall BF, Labherz TB, Rubinstein L, Robertson RD, Sample WF, Sarti D, et al. Chromosome findings in 2500 second trimester amniocenteses. Am J Med Genet 1980;5:345-56.

15. Kim YJ, Ryu HM, Park SY, Kim MY, Han JY, Yang JH, et al. Parental Decisions of Prenatally Detected Sex Chromosome Abnormality. J Korean Med Sci 2002;17:53-7.

16. Balkan M, Kalkanli S, Akbas H, Yalinkaya A, Alp MN, Budak T. Parental decisions regarding a prenatally detected fetal chromosomal abnormality and the impact of genetic counseling: an analysis of 38 cases with aneuploidy in Southeast Turkey. J Genet Couns 2010;19:241-6.

17. Christian SM, Koehn D, Pillay R, MacDougall A, Wilson RD. Parental decisions following prenatal diagnosis of sex chromosome aneuploidy: A trend over time. Prenat Diagn 2000;20:37-40.

18. Meschede D, Louwen F, Nippert I, Holzgreve W, Miny P, Horst J. Low rates of pregnancy termination for prenatally diagnosed Klinefelter syndrome and other sex chromosome polysomies. Am J Med Genet 1998;80:330-4.

19. Shaw SW, Chueh HY, Chang SD, Cheng PJ, Hsieh TT, Soong YK. Parental decisions regarding prenatally detected fetal sex chromosomal abnormality and the impact of genetic counselling: An analysis of 57 cases in Taiwan. Aust N Z J Obstet Gynaecol 2008;48:155-9.

20. Marteau T, Drake H, Reid M, Feijoo M, Soares M, Nippert I, et al. Counselling following diagnosis of fetal abnormality: a comparison between German, Portuguese and UK geneticists. Eur J Hum Genet 1994;2:96-102.

21. Christian SM, Koehn D, Pillay R, MacDougall A, Wilson RD. Parental decisions following prenatal diagnosis of sex chromosome abnormality: a trend over time. Prenat Diagn 2000;20:37-40. 